\title{
Asteroseismic Diagnostics for Semi-Convection in B Stars in the Era of K2
}

\author{
Ehsan Moravveji $\dagger$ \\ Instituut voor Sterrenkunde, KU Leuven, Celestijnenlaan 200D, B-3001 Leuven, Belgium \\ email: Ehsan.Moravveji@ster.kuleuven.be
}

\begin{abstract}
Semi-convection is a slow mixing process in chemically-inhomogeneous radiative interiors of stars. In massive OB stars, it is important during the main sequence. However, the efficiency of this mixing mechanism is not properly gauged yet. Here, we argue that asteroseismology of $\beta$ Cep pulsators is capable of distinguishing between models of varying semi-convection efficiencies. We address this in the light of upcoming high-precision space photometry to be obtained with the Kepler two-wheel mission for massive stars along the ecliptic.
\end{abstract}

Keywords. asteroseismology, stars: oscillations (including pulsations), stars: interiors, stars: evolution, stars: rotation, variables: others

\section{Introduction}

Non-radial pulsation is a common phenomenon among B dwarfs. Early-type B stars widely known as $\beta$ Cep stars - are pulsationally unstable against low-order, low-degree radial and non-radial pressure (p-) and gravity (g-) modes. Their mass ranges from $\sim 8$ to $20 M_{\odot}$ (see Aerts et al. 2010, for details). Contrary to their fully mixed convective cores, the mixing of species in their radiative interior occurs on a long - yet unconstrained time scale. There are several mixing mechanisms that operate (simultaneously) in radiative zones, among which rotational mixing and semi-convection. In this paper, we limit ourselves to slowly-rotating B stars.

The pulsation frequencies of stars are highly sensitive to their internal structure, and can be used as a proxy to test different input physics. Miglio et al. (2008) already showed the effect of extra mixing induced by, e.g., rotation, atomic diffusion, and convective overshooting on the period spacing of g-modes in heat-driven pulsators; their conclusions can be extended to include semi-convection as an extra mixing mechanism.

In the near future, the Kepler two-wheel mission, (hereafter K2, Howell et al. 2014) will provide high-precision space photometry of a handful of late-O and early B-type pulsators in the ecliptic plane. We emphasise that $\mathrm{K} 2$ will conduct pioneering observations, since such space photometry is scarce for massive stars, particularly for objects more massive than $8 M_{\odot}$.

In this paper, we put forward asteroseismic diagnostics to probe semi-convective mixing in massive main-sequence stars. Following on Miglio et al. (2008), we address the possibility of constraining the efficiency of semi-convection in massive stars in light of the upcoming high-precision data to be assembled by the K2 mission and already present in the CoRoT archive.

$\dagger$ Postdoctoral Fellow of the Belgian Science Policy Office (BELSPO), Belgium 

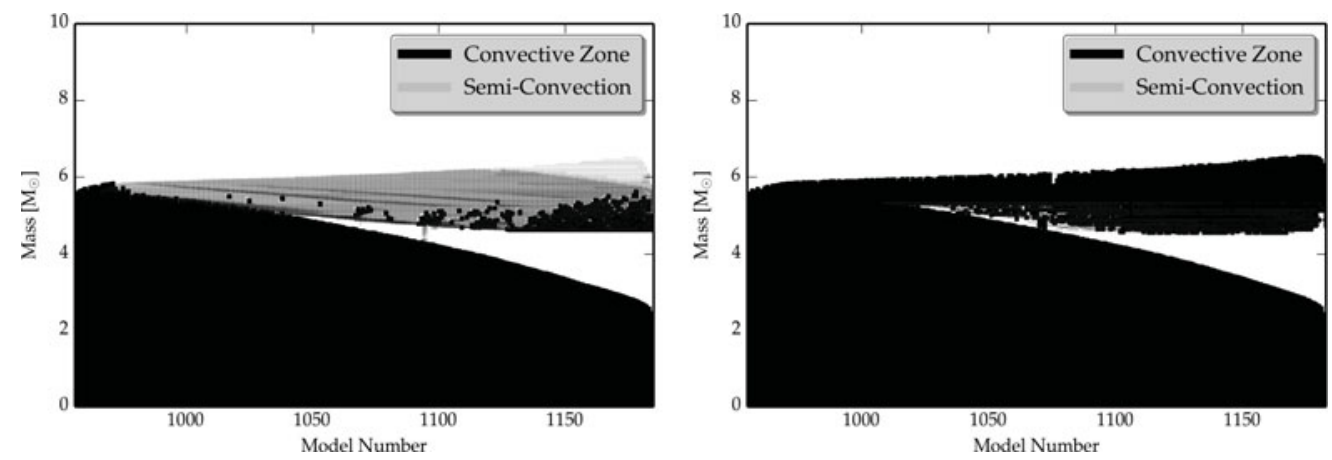

Figure 1. Kippenhahn diagrams showing the evolution of $15 M_{\odot}$ stellar models with $\alpha_{\mathrm{sc}}=10^{-1}$ (left) and $\alpha_{\mathrm{sc}}=1$ (right). The convective zones are shown in blue, and the semi-convective zones are shown in purple.

\section{Semi-Convective Mixing}

Semi-convection is a slow mixing process believed to operate in the chemically inhomogeneous parts of radiative zones, where the g-modes are oscillatory (Schwarzschild \& Härm 1958; Kato 1966; Langer et al. 1983; Noels et al. 2010). In other words, semiconvection acts in those layers of the star where the radiative temperature gradient $\nabla_{\text {rad }}$ takes values in between the adiabatic $\nabla_{\text {ad }}$ and the Ledoux $\nabla_{\mathrm{L}}$ gradients, i.e., $\nabla_{\mathrm{ad}}<$ $\nabla_{\mathrm{rad}}<\nabla_{\mathrm{L}}$. Here, $\nabla_{\mathrm{L}}=\nabla_{\mathrm{ad}}+\varphi / \delta \nabla_{\mu}$, with $\varphi=(\partial \ln \rho / \partial \ln T)_{P, \mu}, \delta=(\partial \ln \rho / \partial \ln \mu)_{P, T}$ and $\nabla_{\mu}=d \ln \mu / d \ln P$. Semi-convection occurs due to the stabilizing effect of the composition gradient $\nabla_{\mu}$ against the onset of convection in the chemically inhomogeneous layers on top of the receding convective core. This mixing process is believed to be present in stars with $M \gtrsim 15 M_{\odot}$ (Langer et al. 1985; Langer 1991). The reason is the increasing effect of the radiation pressure with mass, and the local increase of $\nabla_{\text {rad }}$ with respect to $\nabla_{\text {ad }}$ outside the convective core. Therefore, semi-convection is not expected in intermediate to late B-type stars. For this reason, $\beta$ Cep stars are optimal candidates to investigate if such a mixing can leave observable footprints. The semi-convective mixing is typically described in a diffusion approximation (Langer et al. 1983). See Section 6.2 in Maeder (2009) for an overview of different mixing schemes.

The stellar evolution code MESA (Paxton et al. 2011, 2013) follows the prescription by Langer et al. (1985), where the semi-convective diffusion coefficient $D_{\mathrm{sc}}$ is defined according to Kato (1966) and Langer et al. (1983):

$$
D_{\mathrm{sc}}=\alpha_{\mathrm{sc}} \frac{\kappa_{r}}{6 c_{p} \rho} \frac{\nabla-\nabla_{\mathrm{ad}}}{\nabla_{\mathrm{L}}-\nabla}
$$

where $\kappa_{r}=4 a c T^{3} / 3 \kappa \rho$ is the radiative conductivity, $c_{p}$ the specific heat at constant pressure, and $\rho$ denotes the density. The efficiency of semi-convective mixing is controlled by the free parameter $\alpha_{\mathrm{sc}}$, which determines the length scale and time scale of the vibrational mixing associated to semi-convective zones. The parameter $\alpha_{\mathrm{sc}}$ is not calibrated from observations, Langer et al. (e.g., 1985) favoured $\alpha_{\mathrm{sc}}=10^{-1}$, while later on Langer (1991) preferred $0.01 \leqslant \alpha_{\mathrm{sc}} \leqslant 0.04$. Note that the value of $\alpha_{\mathrm{sc}}$ depends on the choice of the opacity tables and on the numerical scheme to compute $D_{\mathrm{sc}}$ according to Eq. (2.1). We aim at constraining $\alpha_{\mathrm{sc}}$ using K2 data. 


\section{Effect of Semi-Convection on $\beta$ Cep models}

We used the MESA code to calculate four evolutionary tracks for a $15 M_{\odot}$ star with initial chemical composition $(X, Y, Z)=(0.710,0.276,0.014)$ based on Nieva \& Przybilla (2012). We excluded mass loss in all models and considered four values of $\alpha_{\mathrm{sc}}=10^{-6}$, $10^{-4}, 10^{-2}$, and 1 . We used the Ledoux criterion of convection, and made sure that the condition $\nabla_{\mathrm{rad}}=\nabla_{\mathrm{ad}}=\nabla_{\mathrm{L}}$ was satisfied from the convective side of the core boundary (Gabriel et al. 2014); see also Noels (these proceedings). On each evolutionary track, we stored an equilibrium model for a central hydrogen abundance of $X_{c}=0.10$. We subsequently used the GYRE pulsation code (Townsend \& Teitler 2013) to calculate radial $\ell=0$, dipole $\ell=1$ and quadrupole $\ell=2$ mode frequencies in the adiabatic approximation, for each model. We restricted the comparison of the mode behaviour to low-order modes, i.e., $-5 \leqslant n_{\mathrm{pg}} \leqslant+3$, where $n_{\mathrm{pg}}=n_{\mathrm{p}}-n_{\mathrm{g}}$.

Figure 1 shows Kippenhahn diagrams for models with $\alpha_{\mathrm{sc}}=10^{-2}$ (left) and $\alpha_{\mathrm{sc}}=1$ (right). For relatively limited semi-convective mixing (i.e., $\alpha_{\mathrm{sc}}=10^{-2}$ ), an extended semi-convective zone (grey points) develops and continues at roughly the same mass coordinate. On the other hand, for efficient semi-convective mixing (i.e. $\alpha_{\mathrm{sc}}=1$ ) the former zones are identified as convective and an extended intermediate convective zone (hereafter ICZ) develops. In our models, the ICZ encapsulates $\sim 1 \mathrm{M}_{\odot}$. The formation and presence of an ICZ largely impacts the later evolution of the star. From an asteroseismic point of view, models harbouring an ICZ have smaller radiative zones (measured from the surface), hence a smaller cavity for g-mode propagation. As a result, it is expected that the presence of an ICZ, which in turn results from different semi-convective efficiencies, affects the adiabatic frequencies of low-order low-degree p- and g-modes.

A word of caution about matching detected frequencies of unidentified modes from a grid of asteroseismic models is worthwhile to be made here. Figure 2a shows that the frequency of the radial fundamental mode $\ell=0, n_{\mathrm{pg}}=1$ is quite close to that of an $\ell=2, n_{\mathrm{pg}}=-2$ non-radial g-mode. Without robust mode identification — which by itself is intricate - the interpretation of detected pulsation frequencies can be quite misleading. Therefore, the photometric light curves to be assembled by K2 must be complemented with ground-based multi-colour photometry and/or high-resolution spectroscopy to identify at least one of the detected modes. Non-adiabatic computations might help to identify modes, although there are still severe disagreements between the observed modes in OB stars and those predicted to be excited in the sense that we detect many more modes than foreseen by theory. It is therefore safer not to rely on excitation computations when identifying detected frequencies.

Figure 2 a shows the frequencies of $\ell=0,1$ and $2 \mathrm{p}$ - and g-modes for different values of $\alpha_{\mathrm{sc}}$. For better visibility, few modes with identical $\ell$ and $n_{\mathrm{pg}}$ are connected by lines. Clearly, the change in $\alpha_{\mathrm{sc}}$ shifts most of the frequencies to slightly lower/higher values. The frequency difference of the modes with fixed $\ell$ and $n_{\mathrm{pg}}$ increases with decreasing $\alpha_{\mathrm{sc}}$. An important question is whether we are able to capture such subtle differences observationally from the K2 space photometry.

To answer that, we take the model with $\alpha_{\mathrm{sc}}=1$ as the reference model, hence its frequencies are $f_{i}^{(\mathrm{ref})}$. We compare the relative frequency change $\delta f_{i}^{(\mathrm{th})}$ of each theoretical frequency $f_{i}$ with respect to the reference frequency as

$$
\delta f_{i}^{(\mathrm{th})}=\frac{\left|f_{i}^{(\mathrm{ref})}-f_{i}\right|}{f_{i}^{(\mathrm{ref})}} .
$$

Here, $f_{i}^{(\mathrm{ref})}$ and $f_{i}$ are both calculated using GYRE. From an observational point of view, the frequency precision $\Delta f$ depends on the total observation time base $\Delta T$. A 

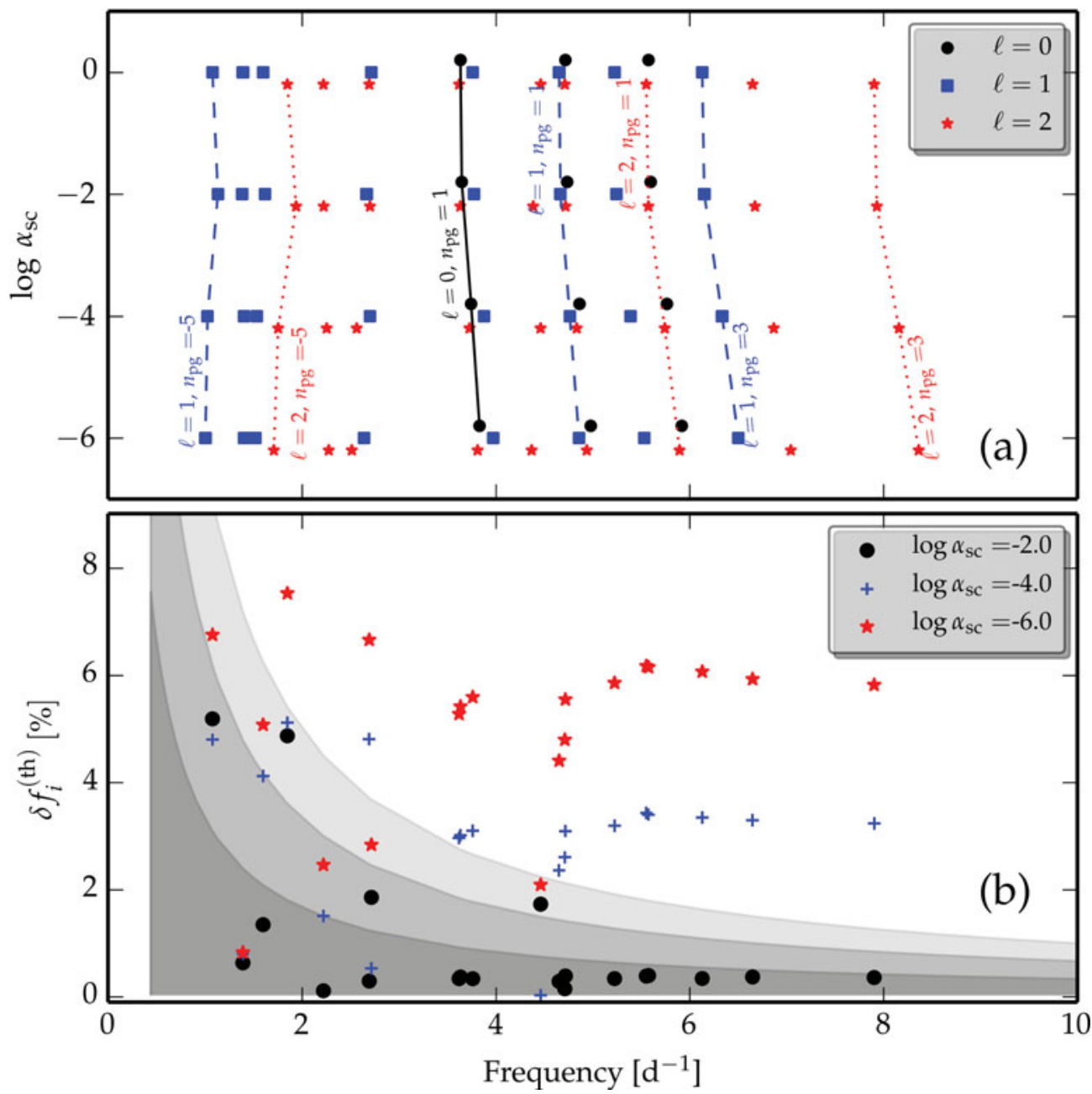

Figure 2. Adiabatic low radial order $-5 \leqslant n_{\mathrm{pg}} \leqslant+3$ frequencies of $15 M_{\odot}$ models with $Z=Z_{\odot}$ and $X_{c}=0.10$. Both panels share the same frequency range on the abscissa. (a) The ordinate is the logarithm of $\alpha_{\mathrm{sc}}$ (Eq. 2.1). Circles are radial $\ell=0$ modes, squares are non-radial dipole $\ell=1$ $\mathrm{p}$ - and g-modes, and stars are non-radial quadrupole $\ell=2 \mathrm{p}$ - and g-modes. (b) Comparison of the relative theoretical frequency change $\delta f^{(\text {th })}$ with the relative observed frequency precision $\delta f^{(\mathrm{obs})}$ as a function of frequency; see Eqs. (3.1) and (3.2). Dark grey, grey and light grey show $1 \sigma, 2 \sigma$ and $3 \sigma$ precision levels, respectively. Circles, plus marks, and stars correspond to models with $\alpha_{\mathrm{sc}}=10^{-2}, 10^{-4}$, and $10^{-6}$, respectively. The reference frequencies are taken from the $\alpha_{\mathrm{sc}}=1$ model. Consult the color version of this figure in electronic format.

conservative estimate by Loumos \& Deeming (1978) is $\Delta f \approx 2.5 / \Delta T$. For K2, the planned $\Delta T$ is approximately 75 days. We also define the relative observed frequency precision $\delta f_{i}^{(\text {obs })}$

$$
\delta f_{i}^{(\mathrm{obs})}=\frac{\Delta f}{f_{i}^{(\mathrm{ref})}},
$$

where $\delta f_{i}^{\text {(obs) }}$ stands for a $1 \sigma$ uncertainty level, and measures the observational frequency precision required to capture the effect of a specific feature - in our case the presence/absence of the ICZ. If by varying a stellar structure free parameter - here 
$\alpha_{\mathrm{sc}}$ - the relative theoretical frequency change is significantly larger than the estimated observational relative frequency precision, i.e. $\delta f_{i}^{(\mathrm{th})} \gtrsim 3 \delta f_{i}^{\text {(obs) }}$, then asteroseismology can constrain the value of that parameter. Note the arbitrary choice of $3 \sigma$ here.

To visualise the probing power of the K2 data, Figure $2 \mathrm{~b}$ shows the relative frequency change $\delta f_{i}^{(\text {th })}$ with respect to the reference model $\left(\alpha_{\mathrm{sc}}=1\right)$ for models with $\log \alpha_{\mathrm{sc}}=-2$ (circles), -4 (plus marks) and -6 (stars), respectively. In the background of the same plot, we show $\delta f_{i}^{\text {(obs) }}$ (dark grey), $2 \times \delta f_{i}^{\text {(obs) }}$ (grey) and $3 \times \delta f_{i}^{\text {(obs) }}$ (light grey), respectively. The distribution of the circles is roughly inside the $1 \sigma$ zone, which makes the distinction between models with $\alpha_{\mathrm{sc}}=1$ and $\alpha_{\mathrm{sc}}=10^{-2}$ quite challenging. However, plus marks that compare $\log \alpha_{\mathrm{sc}}=10^{-4}$ models with those of the reference model, or stars that compare $\log \alpha_{\mathrm{sc}}=10^{-6}$ models with the reference model are more than $3 \sigma$ away from $\delta f^{(\mathrm{obs})}$. For the latter, K2 holds the potential to constrain the efficiency of semi-convection as an extra mixing mechanism, provided that we can find good seismic models of the stars according to the scheme outlined in Aerts (these proceedings) to which we then add the concept of semi-convective mixing.

\section{Conclusions}

Unfortunately, the Kepler mission observed no O-type and early-B stars so far. Thus, we are yet unable to estimate the richness of the frequency spectrum of the K2 light curves for massive dwarfs. The public release of the K2 field 0 light curves is scheduled for September 2014; since there are several OB pulsators on K2 silicon, we will soon be able to gauge the quality of K2 data for massive star asteroseismology.

In our comparisons, we assumed that the $\mathrm{K} 2$ data do not suffer from instrumental effects, hence that $\delta f^{\text {(obs) }}$ depends only on the time base of the K2 campaigns. This is of course an idealised situation. The telescope jitter and drift can easily increase $\delta f^{(\mathrm{obs})}$. Yet, the seismic diagnostic potential stays valid since the $\delta f^{(\mathrm{th})}$ can exceed the $3 \sigma$ level for several modes, and hence the effect of semi-convective mixing can hopefully be detected and studied using K2 light curves.

Based on Figure 2, there is no real preference between modes of different order $n_{\mathrm{pg}}$ and degree $\ell$ in providing asteroseismic diagnostics for semi-convection; in other words, all low-order p- and g-modes possess the same potential to provide a constraint on $\alpha_{\mathrm{sc}}$. The semi-convection free parameter was varied in a broad range, from $10^{-6}$ to 1 , in our exercise. It is of course easier to discriminate between models with large differences in $\alpha_{\mathrm{sc}}$. According to Figure 2b, the distinction between models with $\alpha_{\mathrm{sc}}=10^{-6}$ and 1 is more within reach than distinguishing between models with $\alpha_{\mathrm{sc}}=10^{-2}$ and 1 .

The main message of our work is to emphasis the importance of semi-convection along with the shrinking convective cores in massive OB stars, when the observed frequencies of $\beta$ Cep stars are compared with theoretical frequencies. Consequently, semi-convection - as one of the extra mixing mechanisms in inhomogeneous layers of the stellar radiative interior - could be employed as an extra dimension when modelling stars based on grid calculations coupled to asteroseismic forward modelling (Briquet et al. 2007).

\section{References}

Aerts, C., Christensen-Dalsgaard, J., \& Kurtz, D. W. 2010, Asteroseismology, Astronomy and Astrophsyics Library, Springer Berlin Heidelberg

Briquet, M., Morel, T., Thoul, A., et al. 2007, MNRAS 381, 1482

Gabriel, M., Noels, A., Montalban, J., \& Miglio, A. 2014, ArXiv e-prints

Howell, S. B., Sobeck, C., Haas, M., et al. 2014, PASP 126, 398 
Kato, S. 1966, PASJ 18, 374

Langer, N. 1991, A\&A 252, 669

Langer, N., El Eid, M. F., \& Fricke, K. J. 1985, A\&A 145, 179

Langer, N., Fricke, K. J., \& Sugimoto, D. 1983, A\&\&A 126, 207

Loumos, G. L. \& Deeming, T. J. 1978, Ap\&SS 56, 285

Maeder, A. 2009, Physics, Formation and Evolution of Rotating Stars

Miglio, A., Montalbán, J., Noels, A., \& Eggenberger, P. 2008, MNRAS 386, 1487

Mowlavi, N. \& Forestini, M. 1994, A\& $A$ 282, 843

Nieva, M.-F. \& Przybilla, N. 2012, A\&SA 539, A143

Noels, A., Montalban, J., Miglio, A., Godart, M., \& Ventura, P. 2010, ApשSSS 328, 227

Pápics, P. I., Moravveji, E., Aerts, C., et al. 2014, AESA , in press (arXiv1407.2986)

Paxton, B., Bildsten, L., Dotter, A., et al. 2011, ApJS 192, 3

Paxton, B., Cantiello, M., Arras, P., et al. 2013, ApJS 208, 4

Schwarzschild, M. \& Härm, R. 1958, ApJ 128, 348

Townsend, R. H. D. \& Teitler, S. A. 2013, MNRAS 435, 3406

\section{Discussion}

GEORGY: First a comment: in Georgy et al. (2014), we showed that using the Ledoux criterion improves the fit of the observations of supergiants. However, we did not claim that this is the unique solution.

GEORGY: It seems that your best fit points systematically towards the highest $Z$ you tried for the SPB KIC 10526294. Did you try still higher $Z$ ?

MoravveJI: As shown in Pápics et al. (2014), there exist strong correlations between the different grid parameters - like mass, $Z, X_{\mathrm{c}}$, and overshooting — so that you always find smaller $\chi^{2}$ by increasing $Z$. But we have to stop somewhere, and we have difficulty fine-tuning $Z$ without constraints from spectroscopy.

HIRSCHI: What is the significance of small $\chi^{2}$ differences when the $\chi^{2}$ values are so high?

MorAVVEJI: This means that a limited range of parameters can almost equivalently reproduce the observed frequencies. However, in general when performing seismic modelling of a massive star, the $\chi_{\text {red }}^{2}$ of the best models from a grid including semi-convection and no overshooting is to be compared with that of the best model(s) from a grid taking only overshooting and no semi-convection. This is our aim for the future K2 data.

NoELS: In a $3 M_{\odot}$ star computed with the Schwarzschild criterion, there is no semiconvective zone surrounding the convective core. On the other hand, the use of the Ledoux criterion must give exactly the same extent of convective cores, whatever the mass of the star. In particular, no semi-convective zone is present in models of $3 M_{\odot}$ computed with the Ledoux criterion. I suspect a misplaced convective boundary, which leads you to interpret the layers outside that boundary as semi-convective layers instead of convective ones. Have you checked that the radiative temperature gradient is exactly equal to the adiabatic one at the boundary? This should necessarily be the case, with a radiative temperature gradient lower than the adiabatic one outside the convective core.

MorAVVEJI: We are aware that in the current version of MESA, $\nabla_{\mathrm{rad}}>\nabla_{\mathrm{ad}}$ from the convective side of the boundary when using Ledoux criterion. We have fixed this shortcoming, and then we observe almost identical behaviour of the core boundary whether Schwarzschild or Ledoux criterion is used. Yet, we need to improve some minor aspects of our fix, and then will publish it for use by the community. 